\title{
Chapter 5 \\ Model-Free Control of the Inertia Wheel Inverted Pendulum with real-time Experiments
}

\author{
Ahmed CHEMORI
}

\subsection{Introduction}

The inertia wheel inverted pendulum belongs to the class of underactuated mechanical systems [12] [10] [22]. These systems are characterized by less control inputs than degrees of freedom, meaning that they have at least one unactuated generalized coordinate. Underactuation in these systems has two main sources, the first one is intentionally, i.e. decided in the design stage to minimize the cost, the weight, consumption, etc. The second is non-intentionally, where a fully actuated mechanical system can become underactuated after the deficiency of one or more actuators. Underactuated mechanical systems are characterized by a high nonlinear coupling between actuated and unactuated coordinates [7], and an internal dynamics which is often unstable (i.e. non minimum phase Systems [2]). In the real world many examples of such systems exist, they include, among others, Inverted pendulums [21] [18], under-actuated robot manipulators [6], gymnast robots [31] [23], undersea vehicles [17], aircrafts [26], and some mobile robots [20].

The inertia wheel inverted pendulum [34] is a benchmark for nonlinear control of underactuated mechanical systems. I has attracted the attention of many researchers within control community. Indeed, different control solution have been proposed in the literature these last decades. In [1], strong damping force on the inertia wheel is taken into account in the design of the controller. The stabilization is achieved via nested saturation based controller. [19] solves the limit cycles generation problem on the inertia wheel pendulum using virtual holonomic constraints. Real-time experiments were carried out showing the robustness of the proposed approach. In [27], collocated partial feedback linearization was performed to exhibit the nonlinear core subsystem which is stabilized using an implicit control. The remaining subsystem is stabilized using multiple sliding mode control technique. In our previous work [4] [5], non-collocated partial feedback linearization is used; this gives rise to an unstable internal dynamics, which is stabilized using trajectory optimization and model-based error estimation. In [36] a generalized predictive controller is proposed for the stabilization of the inertia wheel inverted pendulum. In [9], the proposed control solution deals with external disturbance rejection in passivity based control 


\section{Model-Free Control of the Inertia Wheel Inverted Pendulum}

of the inertia wheel inverted pendulum. All the above mentioned techniques require knowledge of the system dynamics and parameters, furthermore only [19] [4] [3] [36] [9] present real-time experiments as a validation on a prototype of the inertia wheel inverted pendulum.

In this chapter, we propose to design a control scheme based on model-free technique to deal with underactuation in stable limit cycle generation. In order to achieve stable limit cycles on both coordinates of the inertia wheel inverted pendulum, we first design a family of p-parameterized periodic trajectories for the pendulum's angle. Those trajectories are then tracked using the control input thanks to a classical model-free controller [15] [8]. Since the system is underactuated and non-minimum phase, a second controller is needed to update the parameter $p$ of the above trajectories in order to deal with the convergence of the internal dynamics of the system. To achieve this control, we propose a second model-free controller using actuated coordinate (inertia wheel) as output and trajectories' parameter $p$ as control input. Note that this control scheme can be easily applied to the stabilization case by carefully choosing appropriate trajectories. Numerical simulations as well as real-time experiments are presented to show the effectiveness of the proposed control scheme and its ability for external disturbances rejection.

The rest of this chapter is organised as follow. In Section 5.2 the experimental platform of the inertia wheel inverted pendulum is presented, with a description of its main components. Then, the proposed control solution is described in section 5.3 for the general case of underactuated mechanical systems. Section 5.4 is devoted to the application of the proposed control scheme to the case of inertia wheel inverted pendulum. Numerical simulations are introduced in section 5.5, where a first validation of the proposed control solution is illustrated. In section 5.6, real-time experimental results are presented and discussed. Finally section 5.7 is devoted to some concluding remarks.

\subsection{Description of the system: Inertia wheel inverted pendulum}

The testbed of the inertia wheel inverted pendulum system is shown in Figure 5.1, with its different components. It consists of three main parts:

- the mechanical part,

- the electronic part, and

- the computer (control PC).

These three main parts will be described in the following. 


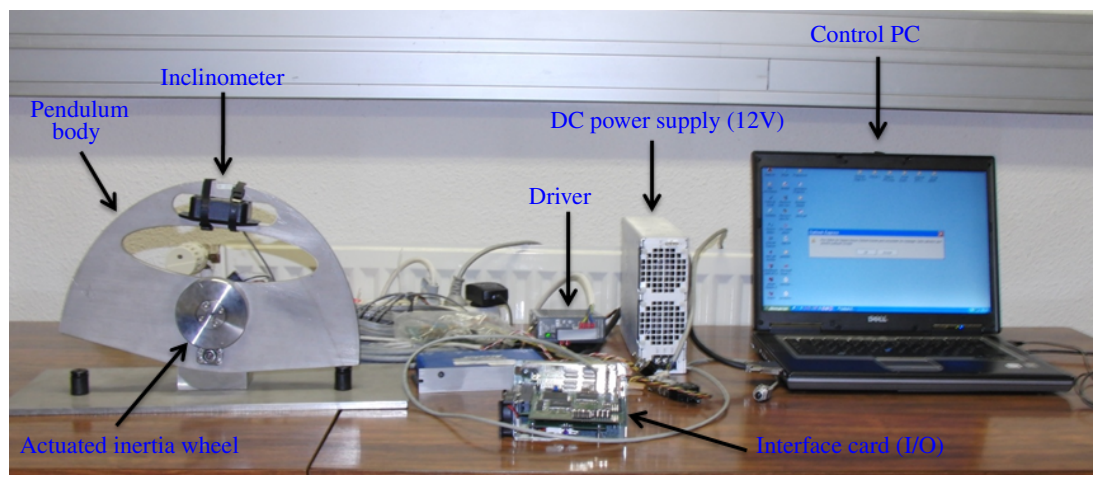

Figure 5.1: View of the testbed of the inertia wheel inverted pendulum system

\subsubsection{Description of the mechanical part of the system}

The mechanical part of the inertia wheel inverted pendulum is shown in Figure 5.2, which consists of an inverted pendulum equipped with an actuated rotating wheel. The joint between the pendulum body and the frame is unactuated whereas the joint between the pendulum body and the wheel is actuated by a Maxon EC-powermax 30 DC motor.

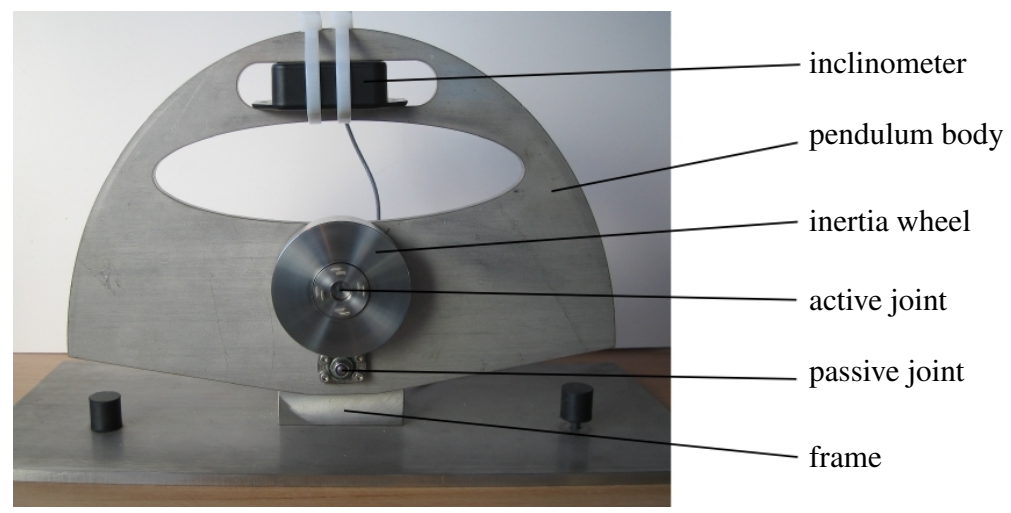

Figure 5.2: View of the mechanical part of the inertia wheel inverted pendulum

The pendulum angle $q_{n a}$ (with respect to the vertical) is constrained to remain within the interval $\left[-10^{\circ},+10^{\circ}\right]$ due to mechanical stops (cf. Figure 5.2). 


\subsubsection{Description of the electronic part of the system}

The actuator of the system is a Maxon EC-powermax 30 DC motor, equipped with an integrated incremental encoder allowing the measurement in real-time of the inertia wheel angular position. To measure the angle of the pendulum with respect to the vertical, the system is equipped with an inclinometer FAS-G of Micro strain. The electronic part of the testbed includes also a MAGMA driver to control the DC motor with a power up to to $200 \mathrm{~W}$, a DC power supply and an I/O interface card. These components are visible on Figure 5.1 of the testbed.

\subsubsection{Description of the control PC and software}

As illustrated in Figure 5.1, the whole system is controlled with a computer equipped with a $2.4 \mathrm{GHz}$ microprocessor. The control software is implemented using C++ language, and the whole system is running under Ardence RTX (Real-Time Extension for Windows) operating system. The computer is the heart of the control system, it is connected to the $\mathrm{I} / \mathrm{O}$ interface card to communicate with the physical system.

\subsection{A Proposed control solution for underactuated systems}

In this section, the proposed control scheme is detailed. The main idea is to develop a control architecture based on model-free control for tracking of reference periodic trajectories while keeping stable the internal dynamics of underactuated mechanical systems.

\subsubsection{Brief background on model-free control}

Model-free control strategy has been initially proposed in [15, 8] resulting in a breakthrough in nonlinear control. This technique is based on previous results on fast estimation and identification of nonlinear signals [16, 24]. The control scheme is based on local linear approximation of the controlled system dynamics which is valid for a small time window. This approximation is updated in an online fashion thanks to a fast estimator. The control law proposed consists in a PID controller augmented with compensating terms provided by online estimation of the system dynamics. The overall controller is also called $i$-PID (standing for intelligent PID). Comparison of such a controller with classical PID controller can be found in [11]. The main advantage of this control strategy is that it doesn't require neither prior knowledge of the system dynamics, nor complex parameters tuning. Consequently, it can be easily applied to controller unknown systems. Some efforts have been made for two particular cases of underactuated systems: the ball and beam [14] (where the dynamics of the beam has not been taken into account) and the Planar Vertical Take Off and Landing (PVTOL) aircraft [28] where the addressed control problem is limited to stabilization. 
For the sake of simplicity, we present in the following model-free control for a Single-Input Single-Output (SISO) nonlinear systems.

\subsubsection{Nonlinear dynamics}

Consider a nonlinear system with unknown dynamics. The input-output behavior of the a system can be expressed in the following general form as an ordinary differential equation (ODE):

$E\left(y, \dot{y}, \ldots, y^{(a)}, u, \dot{u}, \ldots, u^{(b)}\right)=0$

Given that this finite dimensional ODE is smooth enough, it can be approximated for a short time-window by the following simplified model:

$y^{(v)}=F+\alpha u$

The derivation order $v$ and the constant parameter $\alpha \in \mathbb{R}$ can be arbitrarily choosen by the designer. In model-free control litterature, $v$ is generally choosen to be 1 or 2. The non-physical constant $\alpha$ is a design parameter. $F \in \mathbb{R}$ captures all the unknown nonlinearities in the input-ouput behavior and can be compensated in the control law. Since equation (5.2) is valid for a short time window, it must be updated at each sample time. Therefore, the value of $F$ is updated from the measurement of $u$ and $y^{(v)}$ in the following manner:

$[F(k)]_{e}=\left[y^{(v)}(k)\right]_{e}-\alpha u(k-1)$

where $[F(k)]_{e}$ is the estimated value of $F$ at sample time $k$ which will be used for the computation of the control input $u(k) .\left[y^{(v)}(k)\right]_{e}$ is the estimated value of the $v$-th derivative of the output $y$ at discret time $k$ and $u(k-1)$ is the control input previously applied at discret time $k-1$. The value of $F$ can be initialized to 0 at initial time $k=0$.

\subsubsection{The control law}

Given numerical knowledge of $F$ (based on estimation) expressed by equation (5.14), the control input may cancel the unknown nonlinearities and add compensating terms corresponding to a closed-loop tracking of a given reference trajectory $y^{*}(t)$ using a conventional PID controller resulting in an inteligent-PID (often referred to as $i$ PID):

$u=\frac{1}{\alpha}\left(-F+y^{*(v)}+K_{p} e+K_{i} \int e+K_{d} \dot{e}\right)$

where $y^{*(v)}$ is the $v$-th derivative of the reference trajectory $y^{*} . K_{p}, K_{i}, K_{d}$ are the PID feedback gains, $e=y^{*}-y$ is the output tracking error, and $\dot{e}$ is its first timederivative. The tuning of the PID feedback gains can be performed using poles placement techniques since all nonlinearities are supposed to be canceled. It is no longer necessary to perform complex system identification [13, 14]. If $v=1$ the PID controller reduces to a PI controller since the first derivative of output $\dot{y}$ is taken into account in the estimation of $F$ in equation (5.14). 


\subsubsection{Proposed control solution}

In our case, we are interested in tracking of periodic reference trajectories for stable limit cycles generation on under-actuated mechanical systems (mainly known as nonlinear and non-minimum phase) and particularly the inertia wheel inverted pendulum. In order to simplify our presentation of the control problem as well as the proposed solution, let as focus on 1-input 2-degree of freedom underactuated mechanical systems (these are the minimum dimensions for a system to be underactuated) without loss of generality. The dynamics of such systems takes the following lagrangian matrix form [29, 33]:

$M(q) \ddot{q}+H(q, \dot{q})+G(q)=R u$

where $M \in \mathbb{R}^{2 \times 2}$ is the inertia matrix of the system, $q \in \mathbb{R}^{2}$ is the vector of generalized coordinates. $\dot{q}, \ddot{q} \in \mathbb{R}^{2}$ are respectively their first and second time-derivatives. $H \in \mathbb{R}^{2}$ is a vector of centrifugal and Coriolis forces and $G \in \mathbb{R}^{2}$ is a vector of gravitational terms. $u \in \mathbb{R}$ is the control input and $R \in \mathbb{R}^{2 \times 1}$ is a matrix distributing the effects of $u$ on the generalized coordinates of the system. Consider the following partition $q=\left[q_{a}, q_{n a}\right]^{T}$ of the vector of generalized coordinates, where $q_{a}$ is the actuated coordinate and $q_{n a}$ is the unactuated one, then equation (5.5) may be rewritten as:

$m_{11}(q) \ddot{q}_{a}+m_{12}(q) \ddot{q}_{n a}+h_{1}(q, \dot{q})+g_{1}(q)=u$
$m_{21}(q) \ddot{q}_{a}+m_{22}(q) \ddot{q}_{n a}+h_{2}(q, \dot{q})+g_{2}(q)=0$

with :

$$
M=\left[\begin{array}{ll}
m_{11} & m_{12} \\
m_{21} & m_{22}
\end{array}\right] ; \quad H=\left[\begin{array}{l}
h_{1} \\
h_{2}
\end{array}\right] ; \quad G=\left[\begin{array}{l}
g_{1} \\
g_{2}
\end{array}\right]
$$

We suppose that the state of the system is the vector $\left[\begin{array}{lll}q_{a} & q_{n a} & \dot{q}_{a} \\ \dot{q}_{n a}\end{array}\right]^{T}$.

Our goal is to generate stable limit cycles on both actuated and unactuated coordinates of the underactuated mechanical system described by equations (5.6)-(5.7).

\subsubsection{Basic principle of the proposed control scheme}

A family of $p$-parameterized $\tau$-periodic reference trajectories $q_{n a}^{*}(p, \tau, t)$ should be defined for the unactuated coordinate. Those trajectories should have the same boundary conditions for all $p$ values, allowing the controller to switch from one trajectory to another while keeping the overall trajectory smooth. Thanks to the dynamic coupling existing between the actuated and unactuated coordinates of the system, it is possible to control the unactuated coordinate using the control input $u$ (i.e. the torque on the actuated coordinate) which allows those trajectories to be tracked on the unactuated coordinate $q_{n a}$ using the control input $u$.

Indeed, the dynamics (5.6)-(5.7) can be rewritten in a form with a relation between unactuated coordinate and control input [30]. First, equation (5.6) is solved for $\ddot{q}_{a}{ }^{1}$ :

$\ddot{q}_{a}=m_{11}^{-1}\left(-m_{12} \ddot{q}_{n a}-h_{1}-g_{1}+u\right)$.

\footnotetext{
${ }^{1}$ for clarity reasons the dependency in $q$ and $\dot{q}$ of the involved terms is omitted in the adopted notation.
} 
Injecting this solution in equation (5.7) leads to:

$\bar{m}_{2} \ddot{q}_{n a}+\bar{h}_{2}+\bar{g}_{2}=-m_{21} m_{11}^{-1} u$

where $\bar{m}_{2}=m_{22}-m_{21} m_{11}^{-1} m_{12}, \bar{h}_{2}=h_{2}-m_{21} m_{11}^{-1} h_{1}$ and $\bar{g}_{2}=g_{2}-m_{21} m_{11}^{-1} g_{1}$.

Based on the resulting dynamics (5.9), involving a relationship between $\ddot{q}_{n a}$ and $u$, a model-free controller can then be designed to perform the tracking of reference trajectories on unactuated coordinate $q_{n a}$ using the control input $u$. However, since the internal dynamics of the system is unstable (the inertia wheel is a non-minimum phase underactuated mechanical system), even a perfect tracking of these trajectories cannot guarantee the stability of the internal dynamics of the system (i.e. the inertia wheel dynamics). Consequently a second controller is needed to manage the stability of the system's internal dynamics.

Indeed, the closed-loop system dynamics can be obtained by substituting $u$ in equation (5.6) by its value taken from equation (5.7). This requires the invertibility of $-m_{21} m_{11}^{-1}$ and particularly of $m_{21}$. This invertibility constraint is also known as Strong Inertial Coupling [35]. The resulting internal dynamics is then the following:

$\ddot{q}_{a}+m_{12}^{-1} m_{22} \ddot{q}_{n a}+m_{12}^{-1} h_{2}+m_{12}^{-1} g_{2}=0$.

Note that $\ddot{q}_{n a}$ depends on the control input $u$ and therefore on the reference parameterized trajectory and its design parameter $p$. This second controller therefore will acts on the reference trajectory's parameter $p$ (this parameter is considered as the control input to be generated by this second controller) in the aim of keeping the stability of the internal dynamics of the system (i.e. the actuated coordinate is considered as the output to be controlled). In other words a periodic parameterized reference trajectory is tracked on the unactuated coordinate by the first controller, and at the end of each period the second controller chooses the new trajectory parameter $p$ in order to stabilize the actuated coordinate. The chosen parameter $p$ is used in the reference trajectory of the forthcoming cycle. The overall control scheme is illustrated in the block-diagram of Figure 5.3.

\subsubsection{Cyclic reference trajectories generation}

The proposed control framework needs a trajectory generator to provide parameterized reference trajectories $q_{n a}^{*}(p, \tau, t)$ to be tracked on the unactuated coordinate of the system. The proposed trajectories must fulfil some necessary conditions. First of all, they have to be continuous, time-derivable and periodic in order to generate limit cycles. This consideration leads to design oscillatory shaped trajectories which are split up in half period, where we will use symmetry to generate the whole cycle. The parametrization of these trajectories must allow the controller to update the parameter $p$ (which corresponds to the time at which the trajectory $q_{n a}^{*}$ crosses zero) during tracking while the overall trajectory remains smooth. This leads to some initial and 


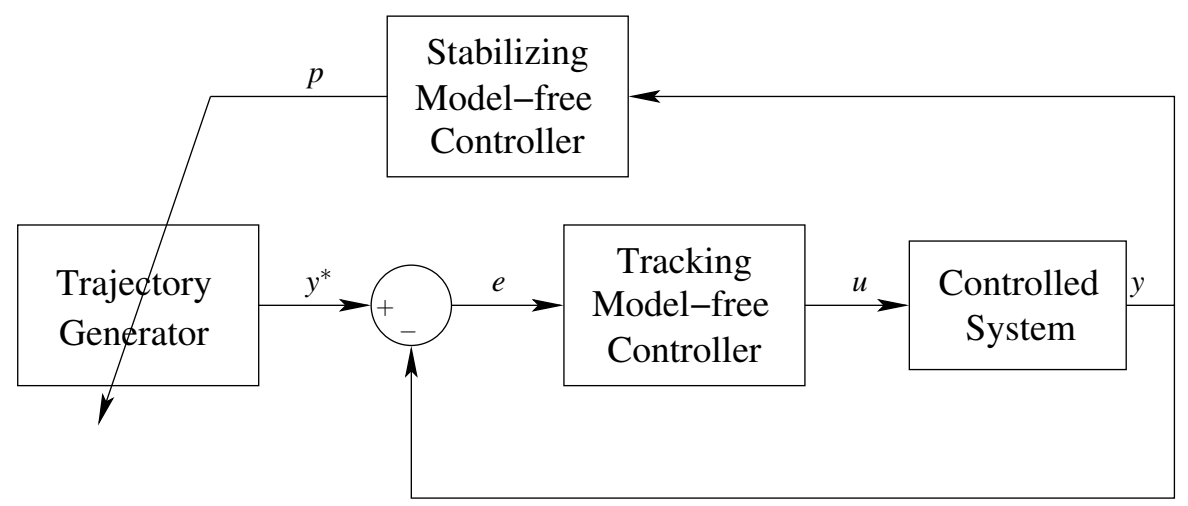

Figure 5.3: Schematic view of the proposed dual model-free controller. The controlled system has as output $y$ and as input $u$. The reference output trajectories $y^{*}$ are generated given a parameter $p$, and tracked by the first model-free controller (called also tracking controller) based on the evaluation of the error $e=y^{*}-y$. The parameter $p$ is updated at each period by the second model-free controller (called also stabilizing controller).

final conditions of each half period part. That is for a given period $\tau$ and amplitude $2 A$ :

$\forall p \in \mathscr{P},\left\{\begin{array}{l}q_{n a}^{*}(p, \tau, 0)=q_{n a}^{*}(p, \tau, \tau)=A \\ q_{n a}^{* a}\left(p, \tau, \frac{\tau}{2}\right)=-A \\ \dot{q}_{n a}^{*}(p, \tau, 0)=\dot{q}_{n a}^{*}\left(p, \tau, \frac{\tau}{2}\right)=\dot{q}_{n a}^{*}(p, \tau, \tau)=0\end{array}\right.$

for some domain $\mathscr{P} \subset \mathbb{R}$ (choosen to keep an oscillatory shape). We propose to use a six-degree polynomial function parameterized with $p$ such that:

$\forall p \in \mathscr{P}, q_{n a}^{*}(p, \tau, t=p)=0$

Figure 5.4 shows normalized reference trajectories (for $\tau=2$ and $A=1$ ) for different values of the parameter $p$ during half a period.

The domain $\mathscr{P}$ is restricted to interval $\left[p_{\min }, p_{\max }\right]$ guaranteeing an oscillatory shape trajectory. It is worth to note, due to the symmetry property of half period trajectory parts, $p_{\min }=1-p_{\max }$.

\subsubsection{The dual model-free controller}

Consider the design of a model-free tracking controller (the first controller in the control framework of Figure 5.3). The first step is to consider the unactuated coordinate nonlinear dynamics (5.9) to be replaced by a local model according to model-free control principle:

$\ddot{q}_{n a}=F_{1}+\alpha_{1} u$ 


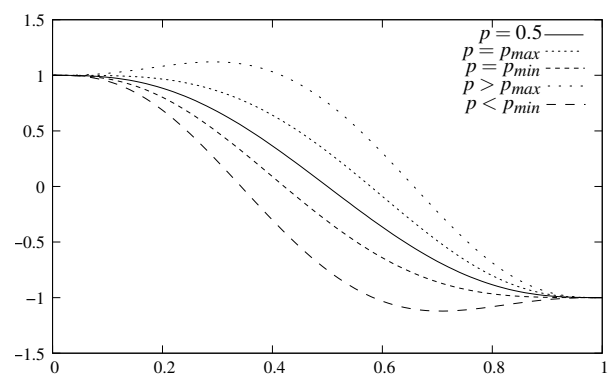

Figure 5.4: Examples of normalized reference trajectories during a half-period for different values of $p$, the period $\tau$ is $2 \mathrm{sec}$ and the amplitude $2 A$ is 2 .

where the constant parameter $\alpha_{1}$ is a control design parameter. The term $F_{1}$ is supposed to capture the nonlinearities in the unactuated coordinate dynamics and is updated at each sample time according to the following equation:

$\left[F_{1}(k)\right]_{e}=\left[\ddot{q}_{n a}(k)\right]_{e}-\alpha_{1} u(k-1)$

where the notation [.] $]_{e}$ states for the estimated value.

The tracking controller is obtained using numerically computed value of $F_{1}$ in (5.14) using the first model-free controller ( $i$-PID) as follows:

$u=\frac{1}{\alpha_{1}}\left(-F_{1}+\ddot{q}_{n a}^{*}(p, \tau, t)+K_{p 1} e+K_{i 1} \int e+K_{d 1} \dot{e}\right)$

where the feedback gains $K_{p 1}, K_{i 1}, K_{d 1}$ can be chosen using poles placement technique based on the assumption that the system's nonlinearities are compensated by the term $F_{1}$. The unactuated coordinate $q_{n a}$ should follow therefore the desired periodic trajectory $q_{n a}^{*}(p, \tau, t)$.

The parameter $p$ in the tracking control input (5.15), is constant over half a period, i.e. $\forall t \in\left[k \frac{\tau}{2}(k+1) \frac{\tau}{2}[, k \in \mathbb{N}\right.$ and is updated at the end of each half period, i.e. at time $k \frac{\tau}{2}, k \in \mathbb{N}$ by a second model-free controller to be designed.

For that purpose let us consider now the dynamics of the actuated coordinate of the system. This dynamics may be approximated by the following local discrete-time model:

$\Delta_{\tau} v_{a}=F_{2}+\alpha_{2} p$

where $\Delta_{\tau} v_{a}=\dot{q}_{a}\left(k \frac{\tau}{2}\right)-\dot{q}_{a}\left((k-1) \frac{\tau}{2}\right)$ is the variation of the velocity of the actuated joint (inertia wheel) $v_{a}=\dot{q}_{a}$ measured between half periods and the constant $\alpha_{2}$ is a control design parameter. According the basic principle of model-free control the value of $F_{2}$ is updated at the end of each half cycle $\left(t=k \frac{\tau}{2}\right.$ for $\left.k \in \mathbb{N}\right)$ as follows:

$\left[F_{2}\left(k \frac{\tau}{2}\right)\right]_{e}=\left[\Delta_{\tau} \dot{q}_{a}\left(k \frac{\tau}{2}\right)\right]_{e}-\alpha_{2} p\left((k-1) \frac{\tau}{2}\right)$ 
Note that continuous dynamics of the actuated coordinate within the half cycle $t=$ $\left[k \frac{\tau}{2}(k+1) \frac{\tau}{2}\right]$ is not taken into account in this local model. The idea is that we only aim at limit cycle generation on this coordinate, therefore it is only required that its evolution should be periodic. In other words, the aim of the second controller is to bring the actuated coordinate as close as possible to a fixed desired state $\left(q_{a}^{d}, \dot{q}_{a}^{d}\right)$ at the end of each half period, ensuring a certain periodicity of its evolution, leading therefore to limit cycles generation. The resulting second model-free controller should updates the trajectory parameter $p$ according to the following control law:

$p=\frac{1}{\alpha_{2}}\left(-F_{2}+K_{p 2}\left(q_{a}^{d}-q_{a}\right)+K_{i 2} \int\left(q_{a}^{d}-q_{a}\right)+K_{d 2}\left(\dot{q}_{a}^{d}-\dot{q}_{a}\right)\right)$

where $K_{p 2}, K_{i 2}, K_{d 2}$ are the PID feedback gains. It is worth to note that since the desired state for actuated coordinate $\left(q_{a}^{d}, \dot{q}_{a}^{d}\right)$ is constant, the $\left(\Delta_{\tau} \dot{q}_{a}\right)^{d}$ term should be zero and is then omitted.

\subsection{Application: the inertia wheel inverted pendulum system}

The testbed of the inertia wheel inverted pendulum is presented in section 5.2. The basis of its mechanical structure is illustrated in figure 5.5.

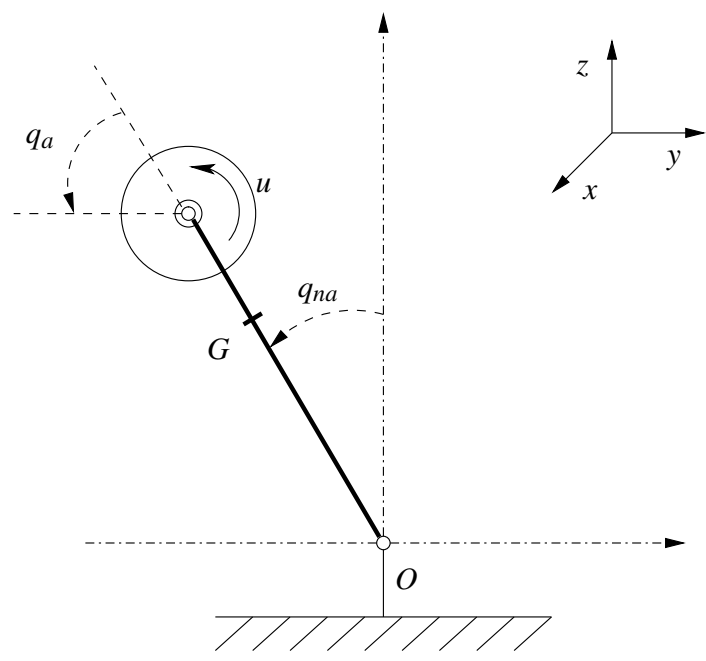

Figure 5.5: Principle schematic view of the inertia wheel inverted pendulum: the joint between the frame and the beam is unactuated (passive joint), while the one between the beam and the inertia wheel is actuated (active joint).

The actuator produces an angular acceleration of the rotating inertia wheel which generates, thanks to the dynamic coupling between coordinates, a torque acting on 
Table 5.1: Description of dynamic parameters of the inertia wheel inverted pendulum

\begin{tabular}{|c|c|c|c|}
\hline Parameter & Description & Value & Unit \\
\hline \hline$m_{1}$ & body mass & 3.228 & $\mathrm{~kg}$ \\
\hline$m_{2}$ & wheel mass & 0.86422 & $\mathrm{~kg}$ \\
\hline$I_{1}$ & body inertia & $3.042 \times 10^{-2}$ & $\mathrm{~kg} \mathrm{~m}^{2}$ \\
\hline$I_{2}$ & wheel inertia & $7.986 \times 10^{-4}$ & $\mathrm{~kg} \mathrm{~m}^{2}$ \\
\hline$l_{1}$ & body CoM position & $6.354 \times 10^{-4}$ & $\mathrm{~m}$ \\
\hline$l_{2}$ & wheel CoM position & $52 \times 10^{-3}$ & $\mathrm{~m}$ \\
\hline
\end{tabular}

the pendulum's passive joint; therefore this passive joint can be controlled through the acceleration of the inertia wheel. The goal of the proposed control solution (as introduced in section 5.3) is to generate stable limit cycles on both coordinates (actuated and unactuated) using only one control input (namely the torque applied to the inertia wheel). The idea is to control the angular position $q_{n a}$ of the pendulum with respect to the vertical by the first controller (tracking controller in Figure 5.3) while the angular velocity of the inertia wheel (system's internal dynamics) is controlled by the second controller (stabilizing controller in Figure 5.3).

\subsubsection{Dynamic modelling of the system}

The mechanical structure of the inertia wheel inverted pendulum is sketched in Figure 5.5. The nonlinear dynamic model [4] of the plant is obtained using Lagrange formulation [32], and is given by:

$$
\begin{aligned}
\left(I_{1}+I_{2}\right) \ddot{q}_{n a}+I_{2} \ddot{q}_{a}-\overline{m l} g \sin q_{n a} & =0 \\
I_{2}\left(\ddot{q}_{n a}+\ddot{q}_{a}\right) & =u
\end{aligned}
$$

where $I_{1}, I_{2}$ are respectively the moments of inertia of the beam and the wheel. $u$ is the torque generated by the motor acting on the inertia wheel. $\overline{m l}=m_{1} l_{1}+m_{2} l_{2}$ with $m_{1}$ and $m_{2}$ being the masses of the pendulum and the inertia wheel. $l_{1}, l_{2}$ are distances from origin $O$ (cf. Figure 5.5) to the gravity centers of the pendulum and the rotating mass (respectively). This model has the general form (5.6)-(5.7) of dynamics of underactuated mechanical systems. The dynamic parameters of the inertia wheel inverted pendulum system are summarized in Table 5.1.

\subsection{Numerical simulations}

Reference trajectories are generated for the unactuated coordinate $q_{n a}$ as presented in section 5.3.2.2. The trajectories amplitude is $2 A=6^{\circ}$ and period is $\tau=2 s$. Numerical simulations have been performed using MATLAB/SIMULINK software of MathWorks. It is worth noting that the dynamic model (5.19)-(5.20) is used to simulate the dynamic behavior of the system, it is not used in the controller design.

The following design parameters were used in the implemented control scheme: 
$\alpha_{1}=-100, \alpha_{2}=50$, the feedback gains of the first controller (tracking controller) are as follows: $K_{p 1}=200, K_{i 1}=0$ and $K_{d 1}=100$. The feedback gains of the second second controller (stabilizing controller) are given by: $K_{p 2}=K_{i 2}=0$ and $K_{d 2}=1$. The sampling frequency is set to $150 \mathrm{~Hz}$ which is a reasonable value for real-time implementation. The desired state for the inertia wheel velocity (controlled by the second controller) is set to $\dot{q}_{a}^{d}=0$.

The obtained simulation results are depicted in Figure 5.6. It is worth to note that a disturbing torque of intensity $0.5 \mathrm{Nm}$ is applied to the pendulum beam at time $t=15$ seconds.

The convergence to a stable limit cycle can clearly be observed on the phase portrait of the pendulum angle $q_{n a}$. The controller reacts immediately to the disturbance as we can see a spike in the control signal (cf. Figure 5.6-(e)). This disturbance induces a deviation from the reference trajectories of the pendulum angle position and velocity which is immediately compensated. Despite the big deviation of the inertia wheel velocity oscillations at the beginning of the simulation due to non zero initial conditions and just after the disturbance, the second controller successfully brings back the inertia wheel velocity trajectory to a periodic trajectory. Indeed, the internal dynamics of the systems converges also to a stable limit cycle. To check the admissibility of the scenario to be implemented on the real system, the motor power admissibility is depicted in Figure 5.6-(g), where it can be seen clearly that the trajectories are inside the admissible region.

\subsection{Real-Time experiments}

Real-time experiments are achieved thanks to the experimental testbed described in section 5.2. It is worth to note that the control design parameters used for these experiments are different from those used in numerical simulation. The first controller parameters are chosen as $\alpha_{1}=-150, K_{p 1}=70, K_{i 1}=0$ and $K_{d 1}=12$. For the Second controller the following parameters are used $\alpha_{2}=70, K_{p 2}=0, K_{i 2}=0$ and $K_{d 2}=1$. Noise filtering was performed through the use of an alpha-beta filter [25] to correctly estimate the pendulum acceleration used in equation (5.14). The following two experimental scenarios have been considered:

- control in nominal case,

- external disturbance rejection.

\subsubsection{Scenario 1: Control in nominal case}

In this first scenario, no external disturbances have been considered. The obtained results for this scenario are depicted in Figure 5.7. The experiment was started by an initial angular position corresponding to a pendulum body at the mechanical stop, introducing an initial position error of $0.125 \mathrm{rad}$ (i.e. $7.16^{\circ}$ ). Despite this initial error, 


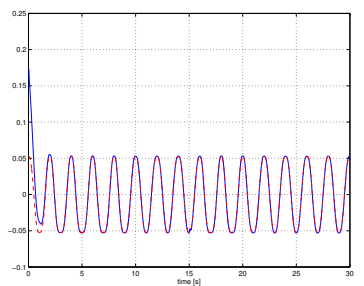

(a) pendulum position $q_{n a}[\mathrm{rad}]$ real position (solid line) and de- $\dot{q}$ sired position (dashed line).

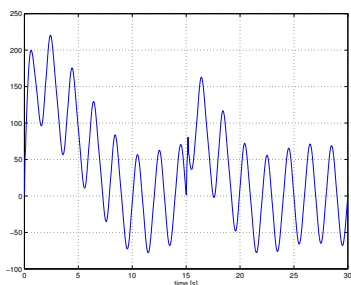

(d) inertia wheel velocity $\dot{q}_{a}\left[\mathrm{rad} \cdot \mathrm{s}^{-1}\right]$.

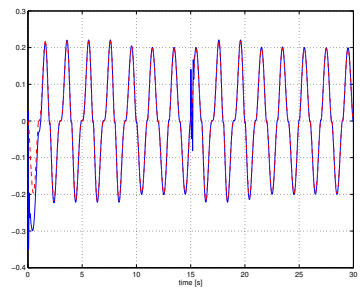

pendulum velocity (ben $\left.\mathrm{s}^{-1}\right]$ teat $\dot{q}_{n a}\left[\mathrm{rad} \cdot \mathrm{s}^{-1}\right]$ real velocity lum $\left(q_{n a}[\mathrm{rad}], \dot{q}_{n a}\left[\mathrm{rad} \cdot \mathrm{s}^{-1}\right]\right)$. (solid line) and desired velocity (dashed line).

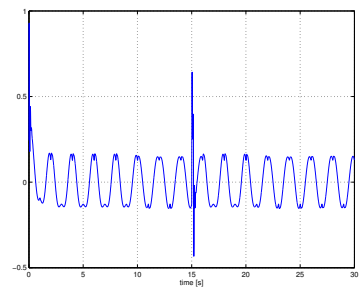

(e) control input $u[\mathrm{~N} \cdot \mathrm{m}]$ versus time.

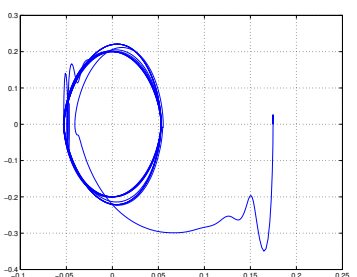

(c) phase portrait of the pendu-

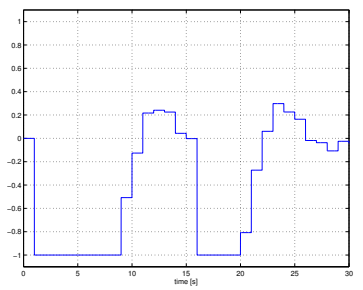

(f) evolution of parameter $p[-]$.

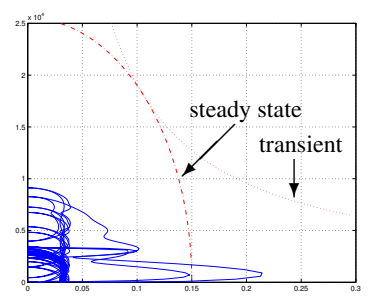

(g) Motor rotationnal speed [rpm] vs Absolute motor torque $[\mathrm{N} \cdot \mathrm{m}]$

Figure 5.6: Simulation results. A punctual external disturbance is introduced as a torque applied at time $t=15 \mathrm{~s}$ to the pendulum beam. 


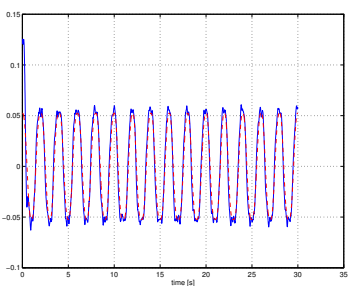

(a) pendulum position $q_{n a}$ real position (solid line) and desired position (dashed line).

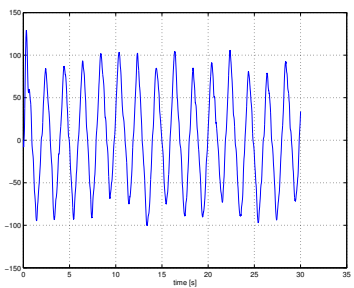

(d) inertia wheel velocity $\dot{q}_{a}$.

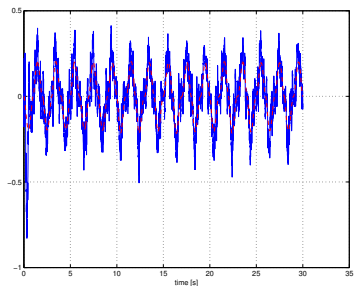

(b) pendulum velocity $\dot{q}_{n a}$ real velocity (solid line) and desire velocity (dashed line).

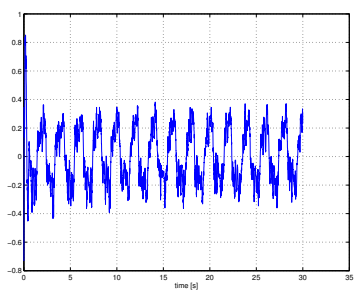

(e) control input $u$ versus time.

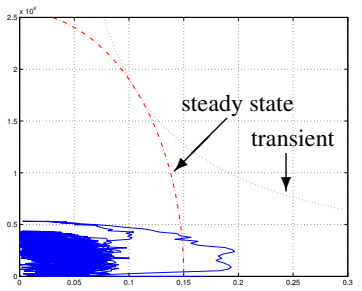

(g) Check of power admissibleregion of the actuator.

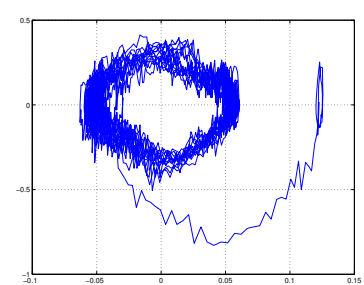

(c) phase portrait of the pendu$\operatorname{lum}\left(q_{n a}, \dot{q}_{n a}\right)$.

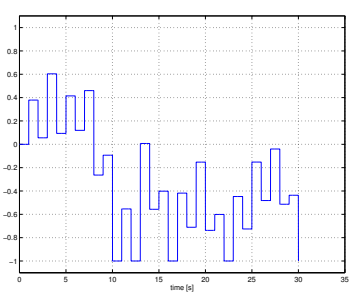

(f) evolution of parameter $p$.

Figure 5.7: Real-time experimental results in nominal case, without external disturbances.

the controller was able to steer rapidly the system towards the periodic reference trajectory as it can be observed on Figure 5.7-(a). The convergence to a stable limit cycle is clearly visible in Figure 5.7-(c). The control inputs are shown in Figure 5.7(e) for the first controller and in Figure 5.7-(f) for the second one. The motor power admissibility is illustrated through the plot of Figure 5.7-(g) showing the evolution of the motor speed (absolute value) versus the its torque (absolute value).

\subsubsection{Scenario 2: External disturbance rejection}

In this experiment, external disturbances are introduced by pushing the pendulum body in a punctual manner as illustrated in Figure 5.8. 


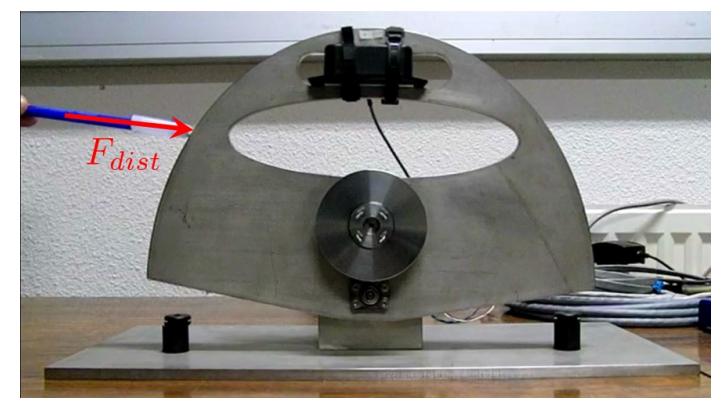

Figure 5.8: Illustration of the external disturbance action applied on the pendulum body during experiments

Tow external disturbances have been introduced at approximately $t_{1}=12 \mathrm{~s}$ and $t_{2}=$ $23 \mathrm{~s}$. The obtained experimental results are plotted in Figure 5.9.

Noise in Measurement is observable on all plots, particularly amplified on the pendulum velocity $\dot{q}_{n a}$ in Figure 5.9(b). Despite this fact, the first controller was able to track the reference trajectory on the pendulum (cf. Figure 5.9(a)). The effect of the introduced external disturbances can clearly be observed on the pendulum trajectories in Figure 5.9(a), and the control input in Figure 5.9(e). The tracking controller brings back rapidly the pendulum position and velocity to their respective reference trajectories. The convergence to a stable limit cycle is clearly visible on the phase portrait of the pendulum shown on Figure 5.9(c). The evolution the inertia wheel velocity is depicted in Figure 5.9(d). Indeed, The introduced disturbances induce a shift in the inertia wheel velocity trajectory cycle. However, thanks to the second controller reaction, which is observable in Figure 5.9(f), the inertia wheel velocity converges back to the limit cycle in few periods. The absolute value of motor velocity versus the generated torque are displayed in Figure 5.9 $(\mathrm{g})$ to check the admissibleregion of the actuator. Notice that the obtained trajectory remains, throughout the experiments, within the admissible region of the actuator's power.

\subsection{Conclusion}

This chapter deals with the control problem of the inertia wheel inverted pendulum. This benchmark belongs to the class of underactuated mechanical systems. The proposed control solution, based on model-free control technique, includes two controllers to deal with stable limit cycle generation. The first controller (called tracking controller) is used to control the angular position of the pendulum (unactuated coordinate), while the angular velocity of the inertia wheel (actuated coordinate) is controlled by the second controller (called stabilizing controller). Indeed, to stabilize 


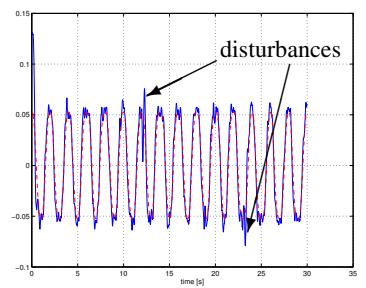

(a) pendulum position $q_{n a}[\mathrm{rad}]$ real position (solid line) and de- $\dot{q}_{r}$ sired position (dashed line).

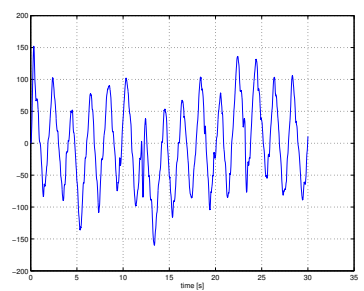

(d) inertia wheel velocity $\dot{q}_{a}\left[\mathrm{rad} \cdot \mathrm{s}^{-1}\right]$.

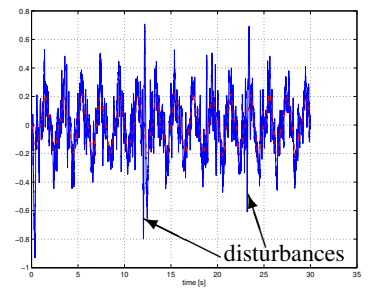

pendulum velocity (c) phase portrait of the pendu-

(c) phase portrait of the pendu-

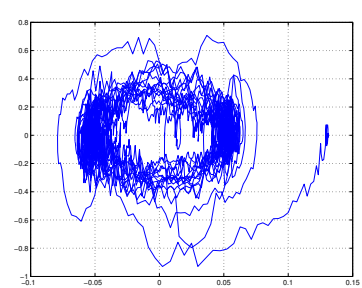
$\operatorname{lum}\left(q_{n a}[\mathrm{rad}], \dot{q}_{n a}\left[\mathrm{rad} \cdot \mathrm{s}^{-1}\right]\right)$. (solid line) and desired velocity (dashed line).
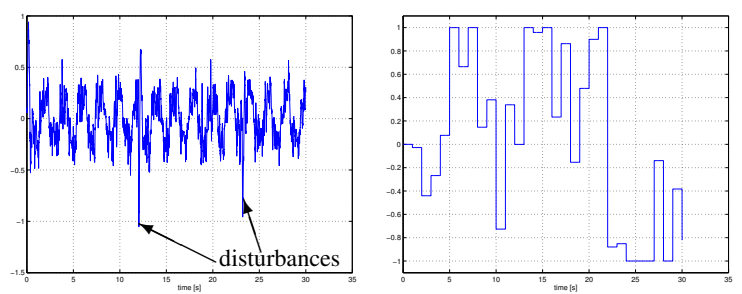

(e) control input $u[\mathrm{~N} \cdot \mathrm{m}]$ versus time.

(f) evolution of parameter $p[-]$.

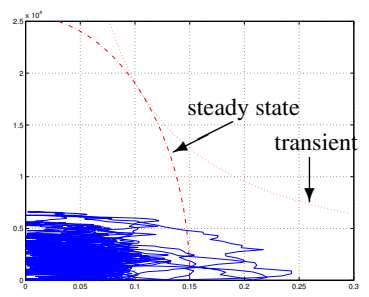

(g) Motor rotational speed [rpm] vs Absolute motor torque $[\mathrm{N} \cdot \mathrm{m}]$

Figure 5.9: Real-time experimental results. Two external disturbances have been introduced at approximately $t_{1}=12 \mathrm{~s}$ and $t_{2}=23 \mathrm{~s}$ on the pendulum body. 
the resulting internal dynamics of the system, a family of $p$-parameterized periodic trajectories is generated and tracked by the first controller, while their parameter $p$ is on-line updated by the second controller in the aim of improving the behavior the internal dynamics (i.e. convergence to a stable limit cycle). To validate the proposed control scheme, numerical simulations as well as real-time experiments are introduced. They show the effectiveness of the proposed control scheme as well as its ability to reject external disturbances. Future work may include the extension of the proposed control scheme to endow it with an automatic adaptive tuning of the parameters of the controllers, as well as its generalization to the case of underactuated systems with more than one degree of underactuation. 
18 Model-Free Control of the Inertia Wheel Inverted Pendulum 


\section{Bibliography}

[1] C.F. Aguilar-Ibanez, O.O. Gutierrez F, and M.S. Suarez Castanon. Stabilization of the strongly damping inertia wheel pendulum by a nested saturation functions. In Proc. of American Control Conference, pages 3434 - 3439, 2008.

[2] S. Andary and A. Chemori. A dual model-free control of non-minimum phase systems for generation of stable limit cycles. In IEEE CDC-ECC, pages 13871392, Orlando, FL USA, 2011.

[3] S. Andary, A. Chemori, M. Benoit, and J. Sallantin. A dual model-free control of underactuated mechanical systems, application to the inertia wheel inverted pendulum. In American Control Conference (ACC'12), pages 10291034, Montréal, Canada, 2012.

[4] S. Andary, A. Chemori, and S. Krut. Control of the Underactuated Inertia Wheel Inverted Pendulum for Stable Limit Cycle Generation. RSJ Advanced Robotics, 23(15):1999-2014, 122009.

[5] S. Andary, A. Chemori, and S. Krut. Estimation-based disturbance rejection in control for limit cycle generation on inertia wheel inverted pendulum testbed. In IEEE/RSJ international conference on Intelligent robots and systems (IROS'09), pages 1302-1307, St. Louis, MO, USA, 2009.

[6] M. Bergerman. Dynamics and Control of Underactuated Manipulators. PhD thesis, Carnegie Mellon University, 1996.

[7] W. Blajer and K. Kolodziejczyk. Modeling of underactuated mechanical systems in partly specified motion. Journal of Theoretical and Applied Mechanics, 46(2):383-394, 2008.

[8] Romain Bourdais, Michel Fliess, Cédric Join, and Wilfrid Perruquetti. Towards a model-free output tracking of switched nonlinear systems. In NOLCOS 2007 - 7th IFAC Symposium on Nonlinear Control Systems, Pretoria, South Africa, 2007.

[9] N. Khraief A. Chemori and S. Belghith. External disturbance rejection in IDA-PBC controller for underactuated mechanical systems: From theory to real-time experiments. In IEEE Multi-Conference on Systems and Control (MSC'14), pages 1747-1752, Antibes, France, 2014. 
[10] A. Choukchou-Braham, B. Cherki, M. Djemai, and K. Busawon. Analysis and Control of Underacuated Mechanical Systems. Springer, London, UK, 2014.

[11] Brigitte D’Andrea Novel, Michel Fliess, Cédric Join, Hugues Mounier, and Bruno Steux. A mathematical explanation via "intelligent" PID controllers of the strange ubiquity of PIDs. In 18th Mediterranean Conference on Control and Automation, MED'10, Marrakech Morocco, 2010.

[12] I. Fantoni and R. Lozano. Non-linear control for underactuated mechanical systems. Springer-Verlag, New York, USA, 2001.

[13] Michel Fliess and Cédric Join. Intelligent PID controllers. In 16th Mediterrean Conference on Control and Automation, Ajaccio, France, 2008.

[14] Michel Fliess and Cédric Join. Model-free control and intelligent PID controllers: towards a possible trivialization of nonlinear control? In 15th IFAC Symposium on System Identification (SYSID 2009), Saint-Malo, France, 2009.

[15] Michel Fliess, Cédric Join, Mamadou Mboup, and Hebertt Sira Ramirez. Vers une commande multivariable sans modèle. In Conférence internationale francophone d'automatique (CIFA 2006), Bordeaux, France, 2006.

[16] Michel Fliess, Cédric Join, and Hebertt Sira Ramirez. Non-linear estimation is easy. Int. J. Modelling Identification and Control, 4:12-27, 2008.

[17] T. I. Fossen. Nonlinear Modelling and Control of Underwater Vehicles. PhD thesis, Norwegian Institute of Technology, 1991.

[18] L. B. Freidovich, P. La Hera, U. Mettin, A. Robertsson, and A. S. Shiriaev. Shaping stable periodic motions of inertia wheel pendulum: Theory and experiments. Asian Journal of Control, 11(5):548-556, 2009.

[19] Leonid Freidovich, Pedro La Hera, Uwe Mettin, Anders Robertsson, Anton Shiriaev, and Rolf Johansson. Shaping stable periodic motions of inertia wheel pendulum: Theory and experiments. Asian Journal of Control, 11(5):548-556, 2009.

[20] S. Ge and F. Lewis. Autonomous Mobile Robots. CRC Press, 2006.

[21] S. Jadlovska and J. Sarnovsky. A complex overview of modeling and control of the rotary single inverted pendulum system. POWER ENGINEERING AND ELECTRICAL ENGINEERING, 11(2):73-85, 2013.

[22] S. Jadlovska and J. Sarnovsky. Modeling and optimal control of nonlinear underactuated mechanical systems Ü a survey. In 13th Scientific Conference of Young Researchers Ü FEI TU of Kosice, pages 32-35, Herlany, Slovakia, 2013.

[23] A. De Luca, R. Mattone, and G. Oriolo. Stabilization of underactuated planar 2r manipulator. Int. J. Robust and Nonlinear Control, 10(4):181-198, 2000. 
[24] Mamadou Mboup, Cédric Join, and Michel Fliess. Numerical differentiation with annihilators in noisy environment. Numerical Algorithms, 50:439-467, 2009.

[25] Robert Penoyer. The alpha-beta filter. C Users J., 11:73-86, 1993.

[26] G. Poulin, A. Chemori, and N. Marchand. Minimum energy oriented global stabilizing control of the PVTOL aircraft. Inter. Jour. of Cont., 80(3):430-442, 2007.

[27] Nadeem Qaiser, Saleh Bin Tariq, Naveed Haq, and Tariq Aziz. A novel nonlinear implicit sliding surface controller design for inertia wheel pendulum. In Proceedings of the 10th WSEAS international conference on Robotics, control and manufacturing technology, ROCOM'10, pages 21-25, Stevens Point, Wisconsin, USA, 2010. World Scientific and Engineering Academy and Society (WSEAS).

[28] Samer Riachy, Michel Fliess, Cédric Join, and Jean-Pierre Barbot. Vers une simplification de la commande non linéaire : l'exemple d'un avion à décollage vertical. In Sixième Conférence Internationale Francophone d'Automatique, CIFA 2010, Nancy, France, 2010.

[29] L. Sciavicco and B. Siciliano. Modeling and control of robot manipulators. McGraw Hill, New York, 1996.

[30] M. Spong. Partial feedback linearization of underactuated mechanical systems. In EEE/RSJ/GI International Conference on Intelligent Robots and Systems, pages 314-321, Munich, Germany, 1994.

[31] M. Spong. The swing up control problem for the acrobot. IEEE Control Systems Magazine, 15(1):49- 55, February 1995.

[32] M. Spong and M. Vidyasagar. Robot Dynamics and Control. John Wiley and Sons, New York, USA, 1989.

[33] Mark W. Spong. Underactuated mechanical systems. In Control Problems in Robotics and Automation. Springer-Verlag, 1998.

[34] Mark W. Spong, Peter Corke, and Rogelio Lozano. Nonlinear control of the inertia wheel pendulum. Automatica, 37:1845-1851, 1999.

[35] M.W. Spong. Partial feedback linearization of underactuated mechanical systems. In Proceedings of the IEEE/RSJ/GI International Conference on IntelligentRobotsand Systems '94, 1994.

[36] N. Touati and A. Chemori. Predictive control for the stabilization of a fast mechatronic system: From simulation to real-time experiments. In International IFAC Symposium on Mechatronic Systems (Mechatronics'13), pages 237-242, Hangzhou, China, 2013. 